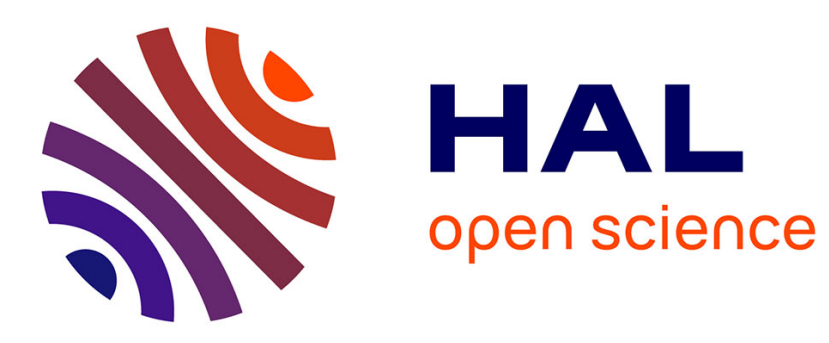

\title{
Service Allocation in Selfish Mobile Ad Hoc Networks Using Vickrey Auction
}

\author{
Jinshan Liu, Valérie Issarny
}

\section{To cite this version:}

Jinshan Liu, Valérie Issarny. Service Allocation in Selfish Mobile Ad Hoc Networks Using Vickrey Auction. 9th International Conference on Extending Database Technology : EDBT 2004, 2004, Heraklion, Cretes, Greece. pp.385-394. inria-00414801

\section{HAL Id: inria-00414801 https://hal.inria.fr/inria-00414801}

Submitted on 10 Sep 2009

HAL is a multi-disciplinary open access archive for the deposit and dissemination of scientific research documents, whether they are published or not. The documents may come from teaching and research institutions in France or abroad, or from public or private research centers.
L'archive ouverte pluridisciplinaire HAL, est destinée au dépôt et à la diffusion de documents scientifiques de niveau recherche, publiés ou non, émanant des établissements d'enseignement et de recherche français ou étrangers, des laboratoires publics ou privés. 


\title{
Service Allocation in Selfish Mobile Ad hoc Networks Using Vickrey Auction
}

\author{
Jinshan Liu and Valérie Issarny \\ INRIA - Rocquencourt, \\ Domaine de Voluceau, Rocquencourt, BP 105, 78153 Le Chesnay Cedex, France \\ \{Jinshan.Liu,Valerie.Issarny\}@inria.fr \\ http: //www-rocq.inria.fr/arles/
}

\begin{abstract}
Incentive scheme for stimulating service provision in Mobile Ad hoc NETworks (MANET) has been under intensive investigation due to its significance to the operation of MANET. This paper applies distributed algorithmic mechanism design and utilizes Vickrey auction for service allocation in mobile ad hoc networks. We show that our method stimulates service provision and achieves desired system-wide service allocation in spite of each agent's selfish behavior, while introducing challenges from the inherent shortcomings of Vickrey auction and characteristics of MANET. We discuss the challenges, the existing solutions for wireline networks and propose a system model for service allocation in MANET.
\end{abstract}

\section{Introduction}

The popularity of light-weight terminals (e.g., handhelds, PDAs and cell phones) with integrated communication capabilities facilitates the ambient intelligence vision of service access anytime, anywhere. Mobile distributed systems that provide access to information and services spread among autonomous devices is of paramount importance to the vision. To realize the above system, cooperation among autonomous devices is a necessity. Unfortunately, cooperative behavior implies resource consumption (e.g., battery), which is not in the interest of the autonomous devices. How to stimulate service provision in mobile ad hoc networks has thus drawn intensive research activities [4, 12, 17, 26].

Not only do we need to stimulate service provision in ad hoc networks, we also need to allocate services in an efficient manner. More specifically, the allocation result should be a social, system-wide choice instead of an individual one. "Service", an instantiated configured system that is run by a providing organization [21], here not only refers to services like packets routing in network layer, but also to services in application layer, e.g., Web services [9]. Current directory-based service repositories (e.g., Jini) require centralized storage scheme and do not address the incentive issues of service allocation. Even with distributed service discovery (e.g., Service Discovery Protocol (SDP) for bluetooth), it is not as trivial as adding a price field in the service description record: (1) one issue is autonomous entities are normally unwilling to reveal their prices, which are considered private; (2) another issue is selecting the least expensive service and 
pay the lowest price does not serve a service most economically 1 . Mechanism design, especially Distributed Algorithmic Mechanism Design (DAMD) [6], is emerging as a suitable approach for solving the above mentioned problem. DAMD addresses the design of incentive compatible mechanisms (i.e., mechanisms that result in desired system-wide outcome from selfish behavior by the system's agents) at tractable computational and communication expense. It lies in the intersection of economics science and computer science.

DAMD is suitable for Mobile Ad hoc NETworks (MANET) because: (1) MANET is distributed by nature; (2) MANET can only afford tractable computational and communication complexity. However, DAMD for MANET is challenged by MANET's resourceconstraints, infrastructureless and unpredictable nature: (1) The inherent mobility of MANET requires the designed mechanism to be robust; (2) Many existing DAMDbased mechanisms for wireline networks assume perfect connectivity between nodes, which is arguable in MANET; (3) Thin devices' limited computational and communication (C2) capability makes $\mathrm{C} 2$-saving a desirable property for mechanisms, as tractable complexity (i.e., polynomial computational complexity) can still be too heavy in many scenarios. Therefore, DAMD employed on wireline networks cannot be applied directly in the context of MANET, which demands specific consideration.

In this paper, we more specifically discuss application of Vickrey auction [23] for service allocation in mobile ad hoc networks. Vickrey auction is a well-known incentive compatible mechanism for solving service and resource allocation problems in multiagent systems in an efficient, distributed and autonomy-preserving manner. Design of algorithmic Vickrey auction falls into DAMD. This paper has three goals: (1) introduce DAMD as a possible tool for designing incentive schemes for MANET; (2) discuss the challenges of applying Vickrey auction in MANET; (3) propose a viable model for service allocation via Vickrey auction in MANET. The paper is organized as follows. The next section introduces mechanism design and various auctions, explains how to apply Vickrey auction as a valuable tool for optimizing service allocation in MANET and outlines the accompanying challenges. Section 3 gives existing solutions in wireline networks for each challenge, and proposes a system model for service allocation via Vickrey auction in MANET. Finally, Section 4 concludes with discussion on future work.

\section{Mechanism Design for Service Allocation in MANET}

The field of mechanism design studies how to design systems so that agents' selfish behavior results in desired system-wide goals. "Agent" here refers to a software entity representing and working for the interests of the host node. However, the game-theory literature on mechanism design neglects computational and communication complexity, which makes mechanism-design approach unpractical in a lot of settings (e.g., Internet). This is addressed by DAMD (Sect. 2.1). Distributed algorithmic auctions, especially Vickrey auction, are a valuable tool for service allocation (Sect. 2.2). After justifying

\footnotetext{
${ }^{1}$ As analyzed in Sect. 2.2 it is actually a first-price, sealed-bid auction, in which bidders' bids are affected by their belief of peers' valuation.
} 
Vickrey auction as the most suitable auction setting for our purpose, we detail the application of Vickrey auction to MANET with confronted challenges (Sect. 2.3).

\subsection{Distributed Algorithmic Mechanism Design}

In essence, Distributed Algorithmic Mechanism Design (DAMD) studies how to design incentive compatible mechanisms with tractable computation (i.e., algorithmic mechanism design [16]) in a distributed setting such as Internet computing [6]. DAMD takes into account communication overhead in addition to the local computational burden on agents.

More formally, consider a distributed system in which there is a set of possible outcomes $\mathcal{O}$ (e.g., result of resource allocation) 2 . A strategy for an agent is a complete contingency plan, i.e., a plan describing what decision the agent should make under each possible situation that might occur. Each of the $n$ autonomous strategic agents 3 has a utility function $u_{i}$ : $\mathcal{O} \rightarrow \mathcal{R}$, where $u_{i} \in \mathcal{U}(\mathcal{U}$ defines the set of utility functions of agents) expresses an agent's preferences over these outcomes. The desired systemwide goals are specified by a Social Choice Function (SCF) $F: \mathcal{U}^{n} \rightarrow \mathcal{O}$ that maps the (actual) utility functions of agents to a particular outcome. However, each agent is usually reluctant to publicize its actual utility function, thus making it impossible to achieve any global goal.

For a given mechanism $M$, let $S$ denote the strategy space of one agent, i.e., a set of strategies that can be taken by the agent, and $C_{M}(u) \subseteq S^{n}$ denote all possible strategy vectors that could reasonably result from selfish behavior. The goal of mechanism design is to define a mechanism $M$ that implements the SCF, i.e., $M\left(C_{M}(U)\right)=F(U)$, for all $U \in \mathcal{U}^{n}$. With such a mechanism qualified as being incentive compatible, selfish behavior by agents will result in desired system-wide outcomes.

In game theory, the strategy that is always in the best interest of one agent, no matter how other agents act, is named dominant strategy. A mechanism with dominant strategy is very desirable for scenarios featuring interactions among autonomous, automated agents, compared to those without dominant strategy: (1) the behavior of agents is much simpler - following the dominant strategy regardless of other agents' behavior; (2) it saves the complex knowledge representation and logic evaluation for counterspeculating how other agents will behave. Thus, it is very desirable to have an incentive compatible mechanism with dominant strategy. The emphasis of mechanism design is put on the implementation of various types of auctions [16], which has been an efficient means for resource allocation [13], service assignment [24] and conflict resolution [5]. In the following, we investigate what kind of auction settings is suitable for our purpose.

\subsection{Auctions}

An auction consists of an auctioneer and potential bidders. The commonly seen auctions include English auction, first-price sealed-bid auction, Dutch auction and Vickrey auction.

\footnotetext{
${ }^{2}$ We rely mostly on [6] for definitions and notation.

${ }^{3}$ Since even random behavior can be considered as one kind of strategy, every agent is a strategic agent, strictly speaking.
} 
English Auction. In English auction, the auctioneer starts with the reserve price and proceeds to solicit successively higher bids from the bidders until no one raises the bid. The highest bidder is the winner and pays the price she bids. The dominant strategy for one agent in English auction is to continuously raise its bid until it wins or it reaches the maximum price it is willing to pay for that item. A noticeable feature of English auction is that it is usually multi-round and the time and communication overhead is proportional to the difference between the starting price and the price at which the item is sold. However, it does allocate the item to the bidder with the highest valuation, who is the only bidder willing to outbid all other bidders.

First-Price, Sealed-Bid Auction. In first-price sealed auction, each bidder submits one bid in ignorance of all other bids to the auctioneer, who determines the highest bid and sells the item to that bidder for the bidding price. This kind of auction can be executed in one-round and thus is communication-saving. However, since each agent's bid is based on her private valuation and prior beliefs of others' valuations, the item is not always awarded to the party who values it most.

Dutch Auction. In Dutch auction, bidding starts at an extremely high price and is progressively lowered until a buyer claims an item by calling "mine". The winner pays the price at the current price. Dutch auction preserves maximal privacy: only the highest bid is revealed. However, like English Auction, it is multi-round, and like first-price, sealed-bid auction, one agent's bid is strategically based on its private valuations and its beliefs of others' valuation.

Vickrey Auction. Similar to the first-price sealed auction, Vickrey auction is sealed and executed in one-round. The highest bidder is the winner, but pays a price that is equal to the second-highest bid [23]. Vickrey auction has a very fundamental feature: the dominant strategy for every bidder is to bid her true valuation 4 . Thus Vickrey auction always rewards the item to the bidder who values it most, i.e., realizes SCF.

Table 1. Various Auction Settings

\begin{tabular}{|l|l|l|l|}
\hline Auction Type & $\begin{array}{l}\text { Communication Com- } \\
\text { plexity }\end{array}$ & $\begin{array}{l}\text { Existence of Dominant } \\
\text { Strategy }\end{array}$ & $\begin{array}{l}\text { Optimal Item } \\
\text { Allocation }\end{array}$ \\
\hline \hline English Auction & Multi-Round & Yes & Yes \\
\hline First-price, Sealed Auction & One-Round & No & No \\
\hline Dutch Auction & Multi-Round & No & No \\
\hline Vickrey Auction & One-Round & Yes & Yes \\
\hline
\end{tabular}

From Table 1 that lists the features of the four previously mentioned auctions, we can see that only Vickrey auction and English auction both have dominant strategy and realize SCF. Furthermore, Vickrey auction only requires single-round execution. Thus,

\footnotetext{
${ }^{4}$ Readers are referred to [22] for the proof.
} 
from the perspective of both economic incentive and communication overhead, Vickrey auction is the best mechanism for service allocation in MANET.

\subsection{Service Allocation via Vickrey Auction in MANET}

It is worth noting that although an auction hosted by a service provider does find the service seeker that values a service most, it does not contribute to our purpose of social service allocation, i.e., serving a service request least expensively, which is essentially to allocate the service request to the service provider that can do so. Therefore, auctions are hosted by service seeking agents, leading to reverse Vickrey auction: the bidder (i.e., service provider) with the lowest instead of the highest bid is the winner.

Bids submitted by service providers are determined by the cost for serving a service, which depends on factors like capabilities of a bidder (e.g., computation power), the load of a bidder (e.g., how many services it has already been serving), the service to be served and some other factors. As the cost reflects suitability of a service provider serving a service better than simply the load, auction-based service allocation is better than non auction-based counterpart because it finds the cheapest way to serve a service via economic payoff. Auction based service allocation also achieves load balancing because heavy load leads to higher bid and thus less possibility of winning the auction.

As mentioned above, service allocation via reverse Vickrey auction in MANET has three desired properties:

1. It has a dominant strategy, thus it is simple to implement.

2. The agents are motivated to bid. The winner gets a payoff which equals to the difference between its valuation and the second lowest bid; the losers lose nothing (i.e., payoff $=0$ ).

3. The service is always allocated efficiently: service (request) is allocated to the provider that serves it least expensively.

Despite Vickrey auction's impressive theoretical properties, Vickrey auction has the following two major shortcomings [19,20]: the fear of dishonest auctioneer and the reluctance of bidders to reveal their true valuation. Since it is sealed, the winner has every reason to doubt the price the auctioneer tells her to pay is actually the second highest price. Thus, fair execution of auctions needs to be guaranteed. Moreover, the valuation of goods or tasks are sensitive and private information that bidders are unwilling to reveal [3, 19].

\section{Design Issues for Service Allocation in MANET}

The design of Vickrey auction for MANET is confronted with challenges posed by the limitation of Vickrey auction and the characteristics of MANET. Below, we investigate the resulting design issues that decompose into: Currency versus Reputation, regarding the remuneration types in auctions for MANET (Sect. 3.1); Fair Execution, on how to solve the problem of dishonest auctioneer, and assure fair execution of Vickrey auction; and Privacy, about how to keep the loser bids private (Sect.3.2). In the end of this section, we propose a system model for carrying out Vickrey auction for service allocation in MANET (Sect. 3.3). 


\subsection{Currency Versus Reputation}

All incentive schemes, including auctions implement a remuneration scheme to incentivize agents' cooperative behavior. Remuneration assumes a specific form that is called renumeration type. Digital currency and reputation are the two most common remuneration types [17].

Digital currency is used to reward cooperative agents; each agent owns some amount of digital currency and the amount is either resident on some security module executed on some tamper resistant hardware [4] or available via some on-line bank service [26]. However, the digital currency scheme does not imply any trust information 5 . Thus, the only way to punish misbehaving agents is to make them lose money (e.g., bidders' put some deposits before the auction [7]).

Alternatively, reputation, which is a perception of an agent's trustworthiness, is resident on other agents based on their previous experience with it. In some systems such as [12], the node can monitor neighbors' activity via promiscuous mode. In that case, experience not only includes direct interaction experiences (e.g., participate in the same auction), but also indirect experiences (e.g., being neighbors but never interact with each other). Experience can even be extended to include other agents' experience via exchange of reputation information [10].

However, because there is no way to bid "reputation" for some service, digital currency is preferred over reputation for a Vickrey auction system. But, reputation can be a supplementary reference for a seller or bidder, e.g., bidders can avoid disreputable auctioneer.

\subsection{Fair Execution and Privacy Preserving of Auctions}

In traditional models of centralized auction (e.g., eBay), there exists a trustworthy party that everybody trusts. It is trivial to conduct an auction in such scenarios, when every bidder can safely submit bids to the trusted party, which determines the winner of the auction. The existence of a trustworthy party is almost impossible in MANET, which makes "fair auction execution" and "privacy preserving" problems even more severe.

The most seen approach for solving the above problems is to transform a single point of trust into a jury of trust; every jury member does not have to be as trustworthy as in traditional models. There are currently two variations of this approach: (1) Jury members can include multiple auctioneers, most of which are assumed to be trustworthy. After each bidder sends shares of their bid to each auctioneer, only a majority of the auctioneers can open the bid with threshold computation (e.g., Verifiable Secret Sharing (VSS) [18]); (2) Jury members can also include a semi-trusted third-party and an auctioneer. Fair execution of auctions and privacy of loser bids are guaranteed if the third-party does not collude with the auctioneer [15], or with any bidder [1].

Executing the above secret sharing protocols requires expensive cryptographic operations, which can hinder the deployment on thin devices. Elliptic Curve Cryptography (ECC) [14] is an alternative to establish public keys, like RSA and DSA. The attraction of ECC is that significantly smaller keys can be used in ECC than other systems like RSA and DSA, but with same level of security (e.g., 160-bit ECC and 1024-bit RSA).

\footnotetext{
${ }^{5}$ You cannot tell one person is trustworthy simply because he/she is rich.
} 
Hence, ECC is preferable for wireless communication due to its bandwidth saving with smaller key size and smaller digital signature [25].

An alternative way to speed up cryptographic operations on mobile devices is to introduce some dedicated hardware for the operations. Compared to the software approach, it is much faster and more physically secure. Low-end chips such as CDL-82 ${ }^{\mathrm{TM}}$ has been planted into PDAs and biometric verification devices6. Additionally, dedicated hardware can also function as "nuglet counter" as mentioned in [4]. The plugging of custom hardware into computation-bounded mobile devices is proving to be a viable approach.

\subsection{Service Allocation System}

Having discussed the issues for applying reverse Vickrey auction for service allocation, we present a system model for conducting such auctions in mobile ad hoc networks. Our approach is essentially based on VSS and uses both remuneration schemes - digital money and reputation. Below is how reverse Vickrey auction for service allocation is carried out after the agents have formed a neighborhood via group management [2].

Service Discovery. For discovering services, we adopt a distributed "pull" multi-hop service discovery scheme. Every service seeking agent can advertise its request, which includes information of service properties (e.g., functional and QoS properties), the auction protocol (Vickrey auction in our case) and an optional list of jury member 7 , by broadcasting it to peers bounded by number of hops. Jury members are chosen by the service seeker according to their reputation of being trustworthy, and are paid by the service seeker for their service 8 .

Reputation Checking. On receiving a service request, each interested service provider first checks whether a jury list is included in the request. If the jury list is absent, potential bidders check whether the service seeker is trustworthy enough for revealing their bids. If the jury list is provided, potential bidders check whether at least two thirds of the members are trustworthy enough for being a jury member because, as to be stated in the next subsection, the auction protocol we use tolerates up to one third of the total shares to be faulty. Therefore, providing a jury list normally increases the possibility of bidding from bidders, which is to the interest of the service seeker.

If reputation checking is passed, potential bidders execute conformance checking with respect to service properties. And, if it does satisfy the request, it estimates the resource consumption for serving such a service. Thereupon, it calculates a bid based on the estimated resource consumption [11], and thus how much it wants to get paid for that service.

Service Allocation. If the jury list is absent, the bidders submit the bids directly to the service seeker and auction is conducted in a traditional centralized way. Alternatively, if

\footnotetext{
${ }^{6}$ Readers are referred to http://www.cdlusa.com/press/press.shtml for further details.

${ }^{7}$ Absence of this list indicates that the auction is to be conducted in a "traditional" mode, i.e., every bidder submits its bid directly to the service seeker.

${ }^{8}$ The allocation of jury member service can also be conducted via Vickrey auction, except that the very first juries for finding jury members may have to be located with the help of some third party.
} 
the jury list is provided, each bidder conducts a secret sharing scheme and submits the shares of the bid to the jury members. The resolution of winner and the price to be paid is done among jury members with an elegant protocol proposed by Harkavy. et. al. [8], which: (i) preserves privacy of bids and anonymity of losers by only revealing the winner and the price to be paid; (ii) tolerates up to one third of missing or faulty shares; and (iii) introduces affordable computation cost. The winner will then get paid with the second lowest bid after completion of service. After each auction, any participant including service seeker, jury members and service provider can update the reputation of any other according to its degree of satisfaction after the auction (e.g., if it is convinced that the auction is fair, or it suspects of any dishonesty). And, the newly update reputation serves as a reference for future encounters.

Note that bidders can have different strategies for submitting bids. An aggressive provider can submit bids to more service requests than it can serve if it wins all the auctions, while a less aggressive provider makes sure that it will be able to serve all the service bids if it wins them all. The strategy very much depends on the current workload of the bidder and the punishment for defaulting a winner bid. As the amount of bids are partially determined by the load of the bidder, it is also affected by the bidder' bidding strategies. The bidders' strategies and their effect on bids is part of our future work.

As for the impact of mobility on our system model, one obvious observation is that our system is robust to leaving of up to one third of the jury members during the auction. However, since our model assumes connectivity among service seekers, jury members and bidders, the auction may be interrupted if mobility disrupts the connectivity.

The overhead of our model lies in the execution of bid resolution protocol. Assume the number of bidders is $n$, number of jury members is $m$, the threshold number is $t$ (i.e., $t$ jury members can not reveal the bids and $3 t<m$ ), and the number of bits to represent a bid is $V$, the communication cost for the auction protocol is $O\left(t^{2} * m * n * \log V\right)$ [8].

\section{Conclusion and Future work}

In this paper, we have discussed issues that arise during design of a Vickrey auction system for service allocation in MANET. The motivation of Vickrey auction for MANET lies in its value for efficient service allocation in a social way. We have shown that although intensive study has been done to solve the problems raised by the limitation of Vickrey auction, additional challenges are confronted due to the infrastructureless and unpredictable nature of MANET. We further have proposed a system model for service allocation via Vickrey auction in MANET.

A possible extension of the current work is to base service allocation on offered QoS together with the amount of bids. One instinctive approach is to base selection on bid divided by client's QoS-dependent utility. But it is problematic because QoS dimensions require normalization since they are in different units [11]. Standard normalization among bids makes bidder's normalized bid dependent on other bids, which makes the existence of dominant strategy doubtful. Additionally, the amount of payment needs careful consideration since the winner is not always the lowest bidder.

In addition to bidders' strategies, as mentioned in the end of last section, our future work also includes adding deposit support for enforcing non-repudiation during the 
auction. This is necessary to guard against winners' defaulting behaviors and enforce honest participation of the auction. Note that the deposit in our work is provided by the service providers (i.e., sellers), contrary to buyers in normal cases. Another extension is to add support for ensuring the fair exchange of service and digital currency between the service seeker and the service provider.

\section{Acknowledgments}

This research is partially supported by the European Commission via IST OZONE (New Technologies and Services for Emerging Nomadic Societies) project? (IST-200030026).

\section{References}

1. O. Baudron and J. Stern. Non-interactive private auctions. In Proc. of Fifth Int'l Conf. on Financial Cryptography (FC), 2001.

2. M. Boulkenafed, J. Liu, D. Sacchetti, and V. Issarny. Group Management in Mobile Ad Hoc Networks: Design, Implementation and Experiments. INRIA Research Report 5060, December 2003.

3. F. Brandt and G. Weiß. Vicious strategies for Vickrey auctions. In Proc. of the Fifth Int'l Conf. on Autonomous Agents, ACM Press, 2001.

4. L. Buttyan and J.P. Hubaux. Stimulating cooperation in self-organizing mobile ad hoc networks. ACM/Kluwer Mobile Networks and Applications, 8, 2003.

5. L. Capra, W. Emmerich, and C. Mascolo. CARISMA: Context-Aware REflective mIddleware System for Mobile Applications. IEEE Transactions of Software Engineering, November 2003.

6. J. Feigenbaum and S. Shenker. Distributed algorithmic mechanism design: Recent results and future directions. In Proc. 6th Int. Workshop on Discrete Algorithms and Methods for Mobile Computing and Communications, ACM Press, 2002.

7. M. Franklin and M. Reiter. The design and implementation of a secure auction service. IEEE Transactions on Software Engineering 5:302-312, 1996.

8. M. Harkavy, J.D. Tygar, and H. Kikuchi. Electronic auctions with private bids. In Proc. of the 3rd USENIX Workshop on Electronic Commerce, 1998.

9. V. Issarny, D. Sacchetti, F. Tartanoglu, F. Sailhan, R. Chibout, N. Levy, and A. Talamona. Developing Ambient Intelligence Systems: A Solution based on Web Services. Journal of Automated Software Engineering, 2004, to appear.

10. J. Liu and V. Issarny. Enhanced reputation mechanism for mobile ad hoc networks. In Proc. of the 2nd Int'l Conf. on Trust Management (i'Trust 2004), March 2004.

11. J. Liu and V. Issarny. QoS-aware service location in mobile ad hoc networks. In Proc. of 2004 IEEE Int'l Conf. on Mobile Data Management, January 2004.

12. S. Marti, T.J. Giuli, K. Lai, and M. Baker. Mitigating routing misbehavior in mobile ad hoc networks. In Proc. of the 6th ACM International Conf. on Mobile Computing and Networking, August 2000.

13. M. McMillan. Selling spectrum rights. Journal of Economic Perspectives, 1994.

\footnotetext{
${ }^{9}$ http://www.extra.research.philips.com/euprojects/ozone
} 
14. V. Miller. Uses of elliptic curves in cryptography. In Proc. of CRYPTO 85, 1985.

15. M. Naor, B. Pinkas, and R. Sumner. Privacy preserving auctions and mechanism design. In Proc. of ACM Conference on Electronic Commerce, 1999.

16. N. Nisan and A. Ronen. Algorithmic mechanism design. In 31st ACM Symp. on Theory of Computing, pages 129-140, 2001.

17. P. Obreiter, B. König-Ries, and M. Klein. Stimulating cooperative behavior of autonomous devices: An analysis of requirements and existing approaches. In Proc. of the 2nd Int'l Workshop on Wireless Information Systems, 2003.

18. T. Pedersen. Non-interactive and information-theoretic secure verifiable secret sharing. In Advances in Cryptology - CRYPTO 1991, pages 129-140, 1991.

19. M.H. Rothkopf, T.J. Teisberg, and E.P. Kahn. Why are Vickrey auctions rare? Journal of Political Economy 98:94-109, 1990.

20. T.W. Sandholm. Limitations of the Vickrey auction in computational multiagent systems. In Proc. of the 2nd Int'l Conf. on Multi-Agent Systems, 1996.

21. C. Szyperski. Component Technology - What, Where and How? In Proc. of the 25th Int'l Conf. on Software Engineering (ICSE'03), 2003.

22. H.R. Varian. Economic mechanism design for computerized agents. In Proceedings of the First Usenix Conference on Electronic Commerce, 1995.

23. W. Vickrey. Counter speculation, auctions, and competitive sealed tenders. Journal of Finance 16:8-37, 1961.

24. N. Vulkan and N.R. Jennings. Efficient mechanisms for the supply of services in multi-agent environments. International Journal of Decision Support Systems, 2000.

25. M.J. Wiener. Performance comparison of public-key cryptosystems. CryptoBytes, Technical Newsletter of RSA Laboratories, 4, 1998.

26. S. Zhong, J. Chen, and Y.R. Yang. Sprite: A simple, cheat-proof, credit-based system for mobile ad-hoc networks. In Proceedings of IEEE Infocom 2003. 